\title{
Software educativo para el trabajo con matrices $^{1}$
}

\author{
Adrian Almaguel Guerra \\ aalmaguelg@udg.co.cu \\ Universidad de Granma \\ Cuba
}

\author{
Denis Alvarez Mora \\ dalvarez1977@yahoo.es \\ Universidad de Granma \\ Cuba
}

\author{
Luis Alberto Pernía Nieves \\ lapernianieves@gmail.com \\ Universidad Técnica de Quevedo \\ Ecuador
}

\author{
Gladys Josefina Mota Pimentel \\ lgadysmota@gmail.com \\ Universidad Politécnica Territorial de Aragua \\ Venezuela
}

\author{
Clemencia Coello León \\ ecoello@uteq.edu.ec \\ Universidad Técnica de Quevedo \\ Ecuador
}

Recibido: Setiembre 15, 2015

Aceptado: Diciembre 14, 2015

Resumen. En el presente trabajo mostramos un software educativo desarrollado por un grupo de investigadores de diferentes universidades con el objetivo de apoyar el proceso de enseñanza aprendizaje de las operaciones con matrices en las carreras de ingeniería. También se muestran los primeros resultados obtenidos con su aplicación.

Palabras clave: software educativo, operaciones matriciales, ingeniería

Abstract. In this paper we present an educational software developed by a group of researchers from different universities in order to support the process of learning of matrix operations in engineering careers. The first results obtained with application are also shown.

KeyWords: educational software, matrix operations, engineering

\footnotetext{
${ }^{1}$ Disponible en: http://www .4shared.com/file/k1UXCBXEce/Matriz.html
} 


\subsection{Introducción}

Desde el surgimiento de las computadoras éstas se nos han presentado como un elemento útil y necesario de la vida cotidiana, su utilización es fácilmente observable en profesiones tan diversas como la medicina, la educación, la ingeniería, la arquitectura, la administración, etc. o en sectores como el gobierno, la industria, la banca o el comercio, lo cual se debe a que estos novedosos artículos tienen como virtud principal procesar con mucha facilidad y a gran velocidad enormes volúmenes de información. En Cuba se hace un gran esfuerzo para aprovechar los beneficios de esta tecnología fundamentalmente en la Educación Superior. Se han desarrollado varios software para apoyar el proceso enseñanza-aprendizaje de muchas materias [8], pero quedan algunas como el álgebra lineal, que aunque cuenta con buenos software estos no cumplen con todas las expectativas [15] pues se limitan solo a mostrar el resultado de las operaciones y no así los pasos que se siguen en cada una de ellas.

Los estudiantes de ingenierías, al menos en Cuba, al enfrentarse por primera vez a las operaciones básicas sobre matrices, les resulta difícil comprender la esencia de los métodos empleados así como los diferentes pasos a seguir [12].Algunas de las principales dificultades que se presentan en los cursos de Álgebra Lineal son: el uso del formalismo, el agobio ante las nuevas definiciones y la pérdida de conexión con lo que los alumnos ya saben de matemáticas [6] . Es cierto que hay libros y materiales muy buenos, con excelentes ejemplos como son: "Álgebra lineal con métodos elementales" [11], "Álgebra lineal y teoría de matrices" [1], "Algebra Lineal" [9], entre otros. Pero la tradición en la enseñanza de las operaciones con matrices muchas veces dificulta la comprensión de los principales conceptos y procedimientos que son de gran utilidad para el ingeniero. Por ello, en muchas ocasiones los profesores llevan a los estudiantes sus propias ideas de cómo usar el aparato matemático y cuáles son los procedimientos más sencillos por cuyo intermedio se pueden dominar los métodos que se necesitan para operar con matrices.

El dominio de los principales conceptos, teoremas y métodos de solución son indispensables, pero adquirir ese dominio es difícil [?]. El departamento de Matemática de la Universidad de Granma, Cuba, en ocasiones utiliza una herramienta para mejorar el aprendizaje de las matrices. Esta herramienta es DERIVE, pero no cumple con las expectativas de nuestra investigación, es decir, no muestra el proceso de desarrollo de las operaciones con matrices.

En el mundo existen muchas otras herramientas que son muy potentes en el trabajo con matrices, entre ellos encontramos MATLAB, MATHEMATICA, WIRIS, CALC 3D PROF, WINMAT, entre los más reconocidos [8].

Esas herramientas sin dudas son muy buenas, sin embargo desde el punto de vista pedagógico tienen un inconveniente; estas herramientas sirven, casi exclusivamente para proporcionar resultados, se le introducen los datos y el ordenador muestra solamente la respuesta. Es decir, su nivel de interactividad con el usuario en su proceso de aprendizaje es bajo, debido a que el estudiante no puede ver el desarrollo de las operaciones.

Estas aplicaciones realizan las operaciones matriciales con tiempos de respuestas excelentes y resultados muy exactos; todo esto resulta muy útil para procesos de ingeniería; es decir, situaciones reales para apoyar a especialistas en estos temas, pero para un estudiante esto resulta insuficiente ya que necesitan herramientas que le permitan comprender y visualizar todo el proceso para desarrollar ha- 
bilidades en el trabajo con matrices. En este trabajo se muestra un software desarrollado en nuestro departamento, así como los resultados alcanzados por dos grupos de estudiantes: uno que recibe la materia utilizando el software y otro que la recibe de la manera tradicional.

\subsection{Software educativo}

En la actualidad existen diversas definiciones de software educativo a las que se han arribado luego de múltiples trabajos de investigación desarrollados a lo largo del tiempo.

La formulación de estas definiciones han surgido por el análisis de ciertas características, tales como:

- Función y finalidad del software

- Modalidad

- Rol del alumno

Enunciaremos, entre tantas, tres definiciones de software educativo de acuerdo a diferentes autores:

- $\quad$ "Son los programas de computación realizados con la finalidad de ser utilizados como facilitadores del proceso de enseñanza y consecuentemente del aprendizaje, con algunas características particulares tales como: la facilidad de uso, la interactividad y la posibilidad de personalización de la velocidad de los aprendizajes" [3].

- $\quad$ "Es un programa o conjunto de programas computacionales que se ejecutan dinámicamente según un propósito determinado. Se habla de software educativo cuando los programas incorporan una intencionalidad pedagógica, incluyendo uno o varios objetivos de aprendizaje" [2].

- $\quad$ "Con la expresión 'software educativo' se representa a todos los programas educativos y didácticos creados para computadoras con fines específicos de ser utilizados como medio didáctico, para facilitar los procesos de enseñanza y de aprendizaje" [10]

En nuestra investigación tuvimos en cuenta la última definición pues engloba a las demás, aunque guardan entre sí gran similitud.

\section{Clasificación del software educativo propuesto}

Uno de los aspectos claves que se debe considerar en el desarrollo de software educativo, es el referido a las características de la interface de comunicación, que a su vez deben coincidir con la teoría comunicacional aplicada y con las estrategias que se desarrollan para el logro de determinados procesos mentales [3]. Estas características permiten una clasificación de los diferentes software educativos. Según esta autora, se puede considerar la siguiente clasificación:

- Tutoriales

- Simuladores

Software educativo para el trabajo con matrices . Adrian Almaguel, Denis Alvarez, Luis A.Pernía Derechos Reservados (C) 2016 Revista digital Matemática, Educación e Internet (http://tecdigital.tec.ac.cr/revistamatematica/) 
- Entornos de programación

- Herramientas de autor

Mientras que Galvin Panqueva [7] realiza la siguiente clasificación de los software educativos:

- Tutoriales

- Sistemas de ejercitación y práctica

- Simuladores

- Juegos educativos

- Sistemas expertos

- Sistemas inteligentes de enseñanza

Teniendo en cuentas las clasificaciones anteriores, el software desarrollado para mostrar el procedimiento de las operaciones con matrices se puede clasificar como una herramienta de autor que presenta un sistema de ejercitación y práctica ya que tiene una finalidad didáctica, la computadora constituye el principal soporte para que el estudiante vea la secuencia de pasos en las operaciones con matrices, es muy fácil de usar y brinda a los estudiantes la posibilidad de mantener una gran interactividad.

\section{Descripción del sistema propuesto}

El sistema propuesto fue implementado en el lenguaje de programación C++ Builder. El sistema permite la entrada de matrices así como la modificación de las mismas para el correcto funcionamiento de las operaciones expuestas. Aquí se introducen todos los datos relacionados con las características de las operaciones que serán procesados (figura 1.1).

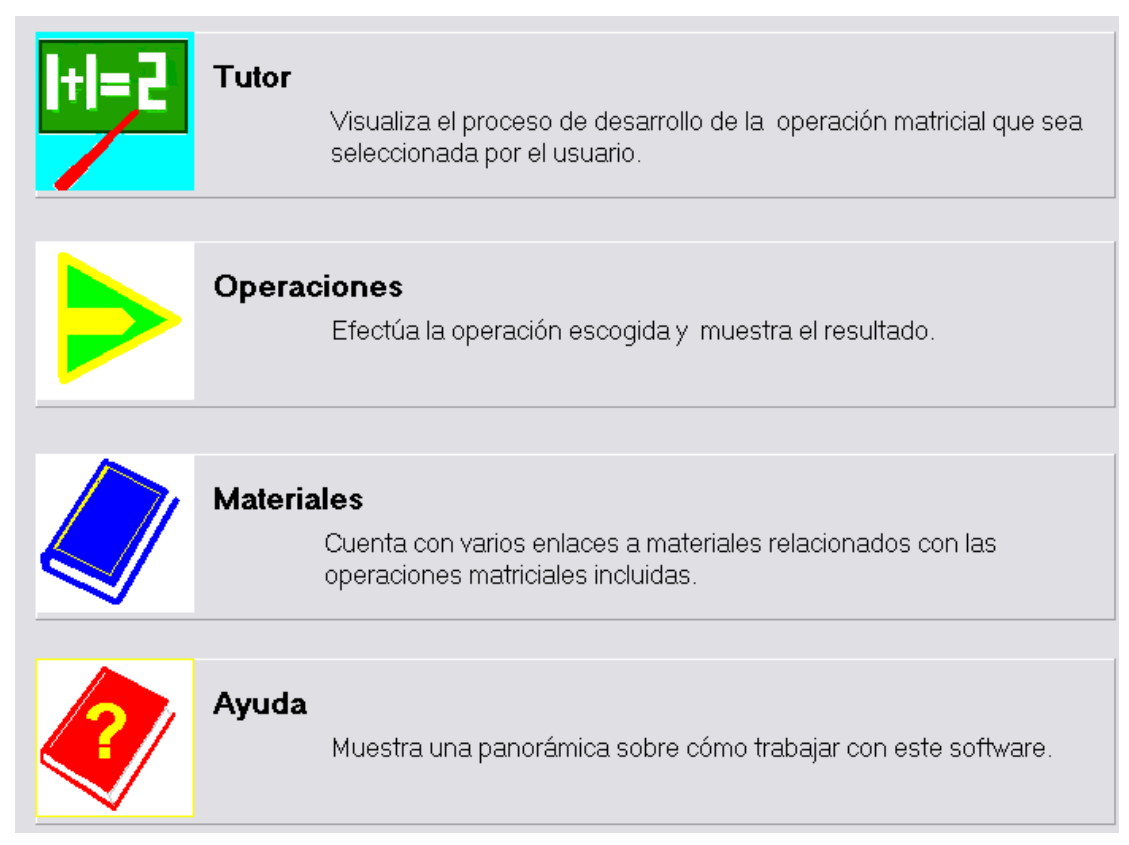


Figura 1.1: Principales opciones del software.

Con los datos antes insertados mediante la opción Tutor se procesa la operación en cuestión y se visualiza el procedimiento de las operaciones mostrando paso a paso como se desarrollan las mismas (figura 1.2).

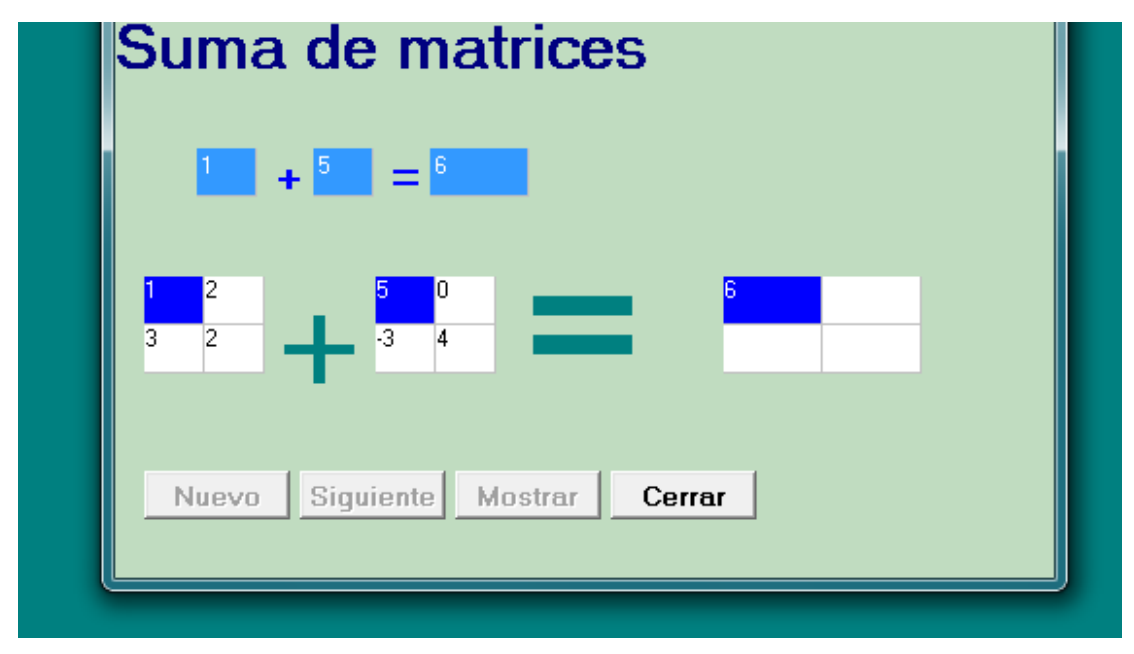

Figura 1.2: Visualización correspondiente a la suma de matrices.

La opción Operaciones realiza la operación escogida y muestra el resultado directamente (figura 1.3)

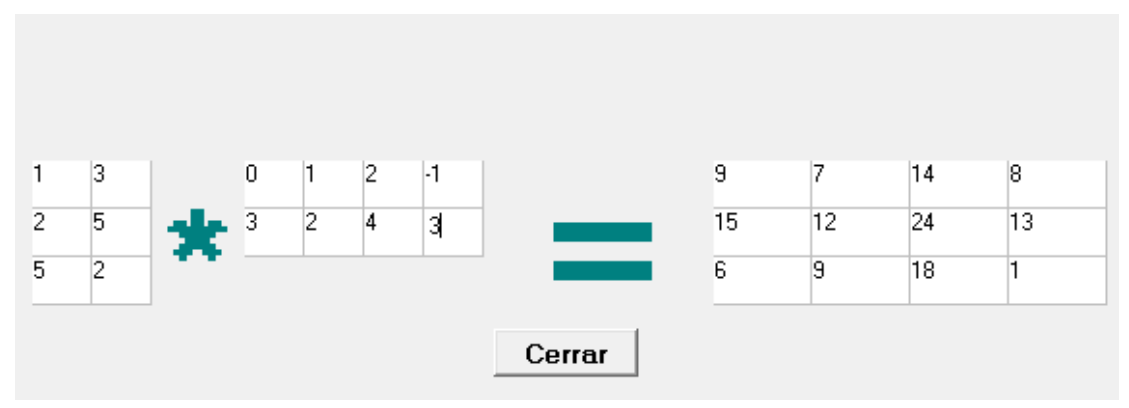

Figura 1.3: Producto de matrices

La opción Materiales permite acceder a materiales propios del sistema donde aparece la parte teórica correspondiente a las operaciones con matrices siendo esta opción novedosa respecto a otros software como el Derive y Matlab.

La opción Ayuda muestra una breve descripción de cómo funciona el software.

\section{Principios de diseño}

El sistema fue diseñado para facilitar de manera sencilla, dinámica y atractiva la visualización de las distintas operaciones matriciales y se logró gracias a la aplicación de algunos principios de diseño:

- Presenta uniformidad e integración 
- La estructura permite cambios

- Proceso de revisión para minimizar los errores conceptuales

\subsubsection{Interfaz de usuario}

La interfaz de usuario es la categoría de diseño que crea un medio de comunicación entre el hombre y la máquina. Además, el diseño identifica los objetos y acciones de la interfaz y crea entonces un formato de pantalla que formará la base del prototipo de interfaz de usuario [13]. Para la interfaz de usuario se tuvieron en cuenta tres reglas :

1. Dar control al usuario.

2. Reducir la carga de memoria al usuario.

3. Consistencia.

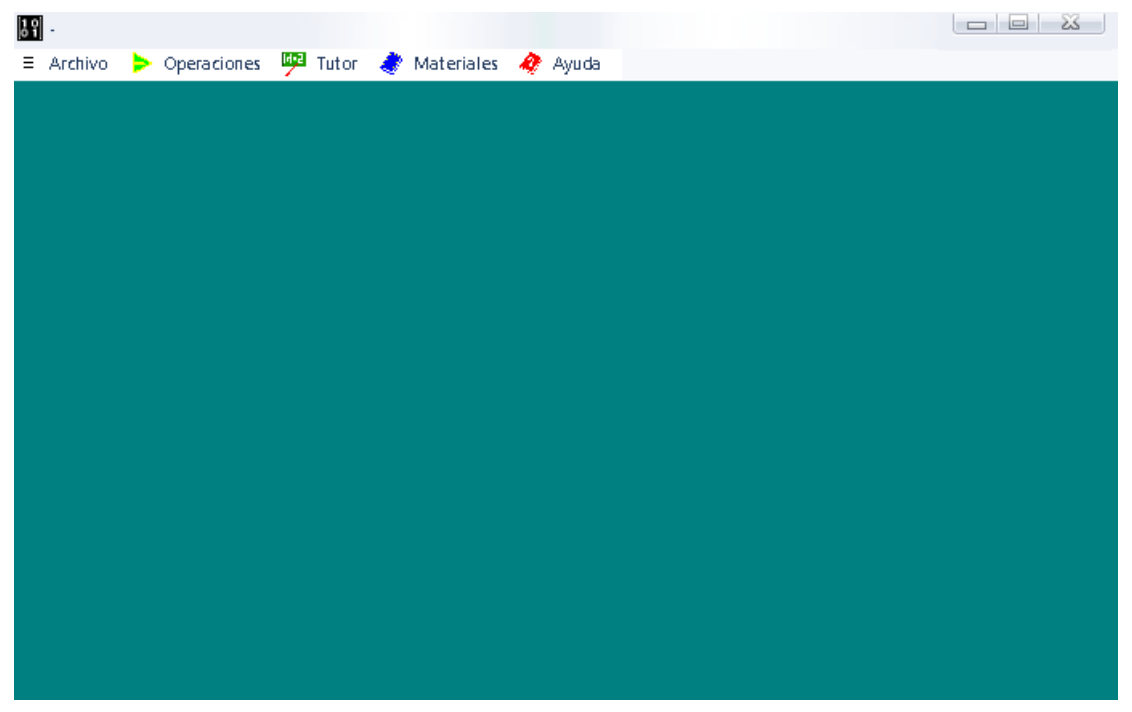

Figura 1.4: La interfaz de usuario.

\subsubsection{Tratamiento de errores}

El sistema garantiza que cuando se produzca un error el usuario sea informado a través de mensajes que aparecerán en la pantalla en el momento que se produzca (figura 1.5) 


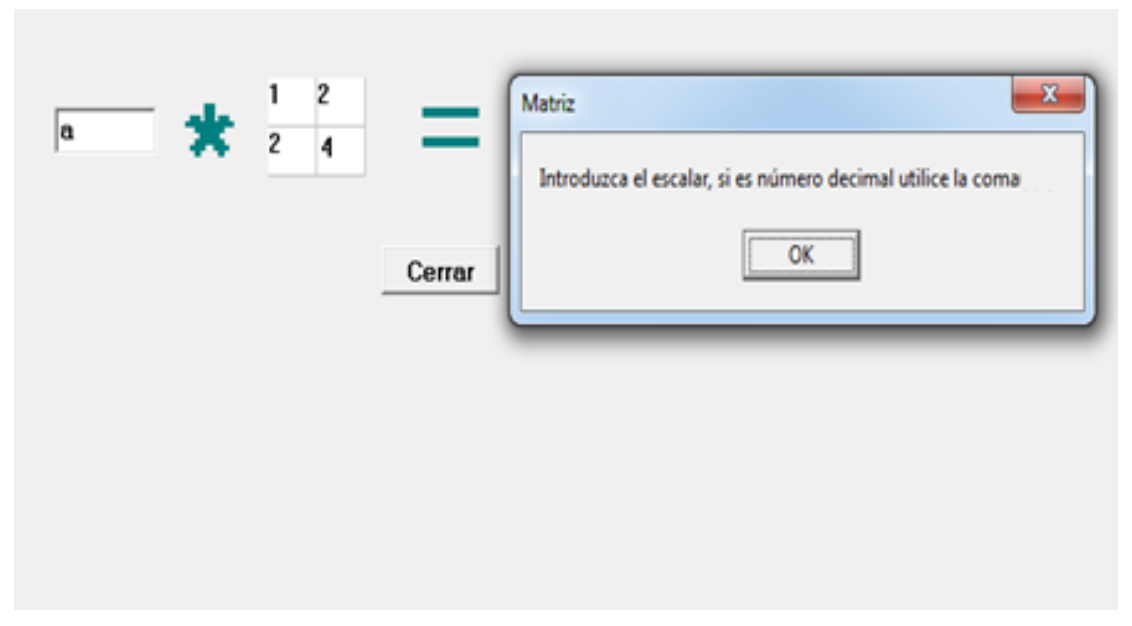

Figura 1.5: Ejemplo de un error cometido y la información brindada por el sistema

\section{Requerimientos}

Para que el sistema trabaje de forma óptima se necesita una computadora que como mínimo tenga las siguientes características:

- Computador con procesador Core Dos Duo

- Disco duro de $160 \mathrm{~GB}$

- 2 Giga Bytes de RAM

- Sistema Operativo Windows

\subsection{Resultados}

Para verificar si la aplicación del software propuesto tenía algún efecto en el proceso de enseñanza aprendizaje en las operaciones con matrices se tomaron 24 estudiantes del grupo de primer año de Ingeniería Informática en el curso 2013-2014, estos estudiantes recibieron el tema de matrices sin utilizar el software, luego en el curso siguiente 2014-2015 se tomaron también 24 estudiantes del grupo de primer año y se le impartió el tema de matrices utilizando la aplicación. Estos últimos 24 estudiantes tenían características similares a los 24 anteriores para así poder conformar 24 parejas de estudiantes donde cada par estuviera formado por estudiantes de años diferentes pero que tuvieran las mismas características en cuanto a los resultados docentes, sexo, edad y nivel educativo de los padres. La materia en los dos cursos fue impartido por mismo profesor el cuál es Licenciado en Matemática con una maestría en Educación. Además tiene 20 años de experiencia en la enseñanza del Algebra Lineal. Los resultados alcanzados por los estudiantes fueron clasificados en Excelente (5), Bien (4) y Regular (3), Mal (2). Luego se recogieron los resultados al aplicarles un examen del contenido de matrices.

Estos resultados se encuentran en una escala de 2 a 5 puntos. El examen aplicado fue similar en complejidad, tanto en un curso como en el otro (Ver anexos 1.41 y 1.42 ). 
Ahora mostraremos los puntajes de cada individuo examinado. Los puntajes de arriba corresponden a los estudiantes que utilizaron el software y lo señalamos con un SI y los de abajo a los estudiantes que no lo utilizaron, que fueron señalados con un NO.

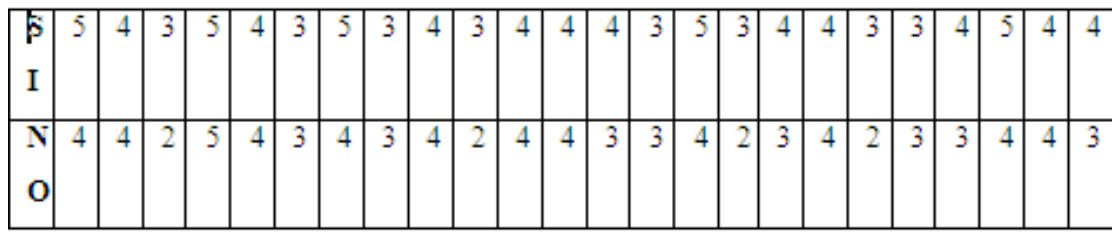

Figura 1.6

Puede afirmarse, con un nivel de significación de 0.05 , que el proceso de enseñanza y aprendizaje de las operaciones con matrices es más favorable, es decir se tiene mejores resultados docentes en los estudiantes que utilizaron el software.

Para corroborar lo anterior se procesaron los datos en el software estadístico SPSS versión 11.5, como estos datos no siguen una distribución normal y además son dos muestras relacionadas por la naturaleza de los estudiantes, se trabajó con la prueba no paramétrica (Prueba de Wilcoxon).

La Hipótesis Nula (Ho) y la Hipótesis Alternativa (Ha), que se definieron para llevar a cabo el estudio fueron las siguientes.

Ho: No hay diferencias en los resultados docentes de los estudiantes que utilizaron el sotware

Ha: Hay diferencias en los resultados docentes de los estudiantes que utilizaron el software

A continuación se muestra el resultado que se obtuvo mediante el software SPSS 11.5.

\section{Ranks}

\begin{tabular}{|ll|r|r|r|}
\hline & N & Mean Rank & Sum of Ranks \\
\hline NO - SI & Negative Ranks & $15^{\mathrm{a}}$ & 13,77 & 206,50 \\
& Positive Ranks & $6^{\mathrm{b}}$ & 4,08 & 24,50 \\
& $3^{\mathrm{c}}$ & & \\
Ties & 24 & & \\
Total & & & \\
\hline
\end{tabular}
a. $\mathrm{NO}<\mathrm{SI}$
b. $\mathrm{NO}>\mathrm{SI}$
c. $\mathrm{SI}=\mathrm{NO}$

Figura 1.7

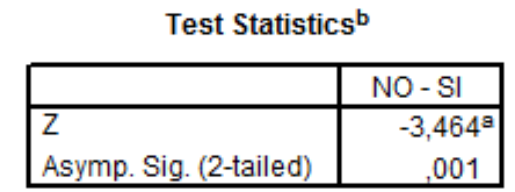

a. Based on positive ranks.

b. Wilcoxon Signed Ranks Test 


\section{Figura 1.8}

En esta prueba se trabaja siempre con la suma de rangos más pequeña y si $P\left(z \leq z_{o b s}\right)<\alpha$ la dócima de hipótesis es significativa, se rechaza la hipótesis nula y se acepta la alternativa que postula la diferencia contraria a la señalada por la suma menor de rangos.

Para el cálculo de la $z$ se emplea la suma de rangos menor y como la dócima es unilateral, el valor de $p$ sería $\frac{0,001}{2}=0,0005<0,05$ por lo tanto la dócima es significativa y se rechaza $H_{0}$, se acepta la alternativa que postula la dirección contraria a la que condujo a la suma de rangos mínima, en este caso $\mathrm{NO}<\mathrm{SI}$, indicando que los estudiantes que utilizaron el software, exhiben mejores resultados docentes, con respecto a los estudiantes que no lo utilizaron.

\subsection{Conclusiones}

El software educativo propuesto puede ser de gran utilidad en el proceso de enseñanza aprendizaje de las operaciones con matrices en las carreras de ingeniería. Los resultados alcanzados por aquellos estudiantes que recibieron la materia utilizando dicha herramienta son superiores a los resultados alcanzados por los estudiantes que recibieron la asignatura en la forma tradicional.

\section{Bibliografía}

[1] Barbolla, P. S. (1998). Álgebra lineal y teoría de matrices. Ed. Prentice- Hall. ISBN 9788483220085

[2] Careaga Butter, M. (2001) Centro de educación y tecnología de Chile. Proyecto Enlaces. Elaborado por. Centro Zonal Sur-Austral. Unidad N 2 Software y su uso pedagógico. Chile.

[3] Cataldi, Z. (2000). Una metodología para el diseño, desarrollo y evaluación de software educativo. Tesis para el Magister de Automatización de Oficinas. ISBN 960-34-0204-2.

[4] Cayley, A. (2008). Algebra de Matrices. Disponible en : http://personal5.iddeo.es/ztt/Tem/T6_ Matrices.htm (consultado el 12 de enero de 2015)

[5] Dhrymes. (2001). Software Educativo. Disponible en : http://es.wikipedia.org/wiki/Software_ educativo (consultado el 21 de marzo de 2015)

[6] Dorier, J., Robert, A., Robinet, J. y Rogalski, M. (2000). The Obstacle of Formalism in Linear Álgebra. En J. Dorier (Ed.), On the Teaching of Linear Álgebra. 85-124.

Software educativo para el trabajo con matrices . Adrian Almaguel, Denis Alvarez, Luis A.Pernía Derechos Reservados (C) 2016 Revista digital Matemática, Educación e Internet (http://tecdigital.tec.ac.cr/revistamatematica/) 
[7] Galvis Panqueva, Á. (1992). Ingeniería de Software Educativo. Universidad de Santa Fe. Bogotá, Colombia. ISBN 958-9057-25-X

[8] García, E. C. R. (2004). Recursos computacionales para la enseñanza aprendizaje de la matemática en la educación superior. Disponible en http://www.monografias.com/trabajos17/computacionmatematicas/computacion-matematicas.shtml (consultado el 28 de marzo de 2012 )

[9] Grossman, S. (1987) Algebra Lineal. Grupo Editorial Iberoamerica, S.A de C.V. México. ISBN 968-7270-39-X

[10] Marqués, P. (1996). El software educativo. Universidad Autónoma de Barcelona. http: //www.Imi.ub.es/te/any96/marques_software/. (Consultado en 1-2012)

[11] Merino, E. S. (2006). Algebra lineal con métodos elementales. Ed. Thomson 2006. ISBN 84-9732-481-1

[12] Mola, C (2012). Estudio sobre la comprensión del álgebra lineal en los estudiantes de ciencias técnicas en la universidad de Camagüey. Revista Pedagogía Universitaria. Vol XXII.No 5.

[13] Presman. (2005). Ingeniería del Software (Vol. 1). Mc. Graw Hill 2002 (ISBN 84-481-3214-9)

[14] Rodríguez, C. Diagnóstico de las dificultades de la enseñanza-aprendizaje en un curso de Álgebra Lineal. XIII Conferencia Iberoamericana de Educación Matemática. Recife, Brasil.

[15] Torres, R. C. (2007). "Enseñanza de las Matemáticas". Disponible en http://www.monografias.com/ trabajos29/ensenanza-matematicas-basada-nuevas-tic/ensenanza-matematicas-basadanuevas-tic.shtml (consultado el17 de abril de 2015) 


\title{
Anexos
}

\section{Anexo 1}

\author{
Universidad de Granma, Cuba \\ Primera evaluación parcial de Algebra Lineal \\ Primer año de Ingeniería Informática \\ Curso 2013-2014
}

\section{Cuestionario}

1. Dada la siguiente matriz $A=\left(\begin{array}{ccc}8 & 1 & 1 \\ 1 & 2 & 2 \\ 1 & 16 & 1\end{array}\right)$ calcular $A^{2}+2 A+I_{3}$

2. Dadas las matrices $A=\left(\begin{array}{ccc}5 & 2 & 1 \\ 1 & 4 & 5 \\ 2 & 10 & 1\end{array}\right)$ y $B=\left(\begin{array}{ll}2 & 1 \\ 2 & 2 \\ 1 & 5\end{array}\right)$ calcular $B^{t} A^{t}$

3. Calcular la inversa de la matriz $\left(\begin{array}{ccc}-1 & 2 & 1 \\ -2 & 5 & 4 \\ -1 & 2 & 2\end{array}\right)$

4. Calcular el siguiente determinante $\left|\begin{array}{ccc}9 & 2 & 2 \\ 27 & 7 & 7 \\ 18 & 5 & 4\end{array}\right|$ 


\section{Anexo 2}

Universidad de Granma, Cuba

Primera evaluación parcial de Algebra Lineal

Primer año de Ingeniería Informática

Curso 2013-2014

\section{Cuestionario}

1. Dada la siguiente matriz $A=\left(\begin{array}{ccc}6 & 2 & 1 \\ 1 & 4 & 4 \\ 2 & 12 & 1\end{array}\right)$ calcular $A^{2}+2 A+I_{3}$

2. Dadas las matrices $A=\left(\begin{array}{ccc}6 & 2 & 1 \\ 1 & 4 & 4 \\ 2 & 12 & 1\end{array}\right)$ y $B=\left(\begin{array}{ll}2 & 1 \\ 2 & 2 \\ 1 & 4\end{array}\right)$ calcular $B^{t} A^{t}$

3. Calcular la inversa de la matriz $\left(\begin{array}{ccc}-1 & 2 & 1 \\ -2 & 5 & 5 \\ -1 & 2 & 2\end{array}\right)$

4. Calcular el siguiente determinante $\left|\begin{array}{ccc}7 & 2 & 4 \\ 22 & 8 & 16 \\ 14 & 5 & 8\end{array}\right|$ 